PROCEEDINGS OF THE

AMERICAN MATHEMATICAL SOCIETY

Volume 135, Number 7, July 2007, Pages 2059-2064

S 0002-9939(07)08748-5

Article electronically published on February 2, 2007

\title{
A REDUCTION THEOREM FOR THE TOPOLOGICAL DEGREE FOR MAPPINGS OF CLASS $(S+)$
}

\author{
J. BERKOVITS
}

(Communicated by Jonathan M. Borwein)

\begin{abstract}
The reduction theorem for the Leray-Schauder degree provides an efficient tool to calculate the value of the degree in a suitable invariant subspace. We shall prove how the calculation of the value of the topological degree for a mapping of class $\left(S_{+}\right)$from a real separable reflexive Banach space $X$ into the dual space $X^{*}$ can be reduced into the calculation of degree of mapping from a closed subspace $V \subset X$ into $V^{*}$. Since the Leray-Schauder mappings are acting from $X$ to $X$ and we are dealing with mappings from $X$ to $X^{*}$, the standard 'invariant subspace' condition must be replaced by a less obvious one.
\end{abstract}

\section{INTRODUCTION}

The classical reduction theorem for the Leray-Schauder degree can be stated as follows (see [7, 8], [10, for instance).

Theorem 1.1. Let $Y$ be a real Banach space, $G \subset Y$ an open bounded set and $F=I-C: \bar{G} \rightarrow Y$ a Leray-Schauder type map, i.e., $C$ is compact. Assume that $0 \notin F(\partial G)$ and $V \subset Y$ is a closed subspace such that

$$
(I-F)(\bar{G}) \subset V \text {. }
$$

Then

$$
d_{L S, Y}(I-C, G, 0)=d_{L S, V}\left(\left.(I-C)\right|_{V}, G \cap V, 0\right) .
$$

Above we have denoted the Leray-Schauder degrees in $Y$ and $V$ by $\mathrm{d}_{L S, Y}$ and $\mathrm{d}_{L S, V}$, respectively. Note that by condition (1.1) $F(\bar{G} \cap V) \subset V$, which justifies speaking about the 'invariant subspace' condition. In fact, condition (1.1) says more than that. An important consequence of condition (1.1) is that $F^{-1}(0) \cap G \subset V$ and hence no solutions were lost in the transition to the smaller space. Note that in case $V \cap G=\emptyset$ both degrees are zero and the theorem is trivially valid. Some extensions of Theorem 1.1 are known; see 2, 7. However, the common feature of all extensions so far is that the domain and range of the mappings involved belong to the same space. This is natural in view of the 'invarant subspace' condition.

Let $X$ be a real separable reflexive Banach space with dual space $X^{*}$ and with continuous pairing $\langle\cdot, \cdot\rangle$ and norm $\|\cdot\|$. We can assume without loss of generality that $X$ and its dual space $X^{*}$ are locally uniformly convex. Recall that for a real

Received by the editors March 2, 2006.

2000 Mathematics Subject Classification. Primary 47H11, 47J05.

Key words and phrases. Topological degree, class $\left(S_{+}\right)$, reduction theorem.

(C)2007 American Mathematical Society Reverts to public domain 28 years from publication 
reflexive Banach space $X$ there exists an equivalent norm such that $X$ equipped with that norm is locally uniformly convex [7]. As a consequence, the induced dual norm in $X^{*}$ also is locally uniformly convex. The norm convergence in $X$ and $X^{*}$ is denoted by $\rightarrow$ and the weak convergence by $\rightarrow$, respectively. We recall that a mapping $F: X \rightarrow X^{*}$ is

- bounded, if it takes any bounded set into a bounded set;

- demicontinuous, if $u_{j} \rightarrow u$ implies $F\left(u_{j}\right) \rightarrow F(u)$;

- of class $\left(S_{+}\right)$, if for any $\left(u_{k}\right) \subset X$ with $u_{k} \rightarrow u, \lim \sup \left\langle F\left(u_{k}\right), u_{k}-u\right\rangle \leq 0$, it follows that $u_{k} \rightarrow u$.

Mappings of monotone type, e.g. pseudomononone mappings and mappings of class $\left(S_{+}\right)$, are widely used in the study of partial differential operators of generalized divergence form. These classes were introduced in the 1970s by Minty, Skrypnik, Browder, Brezis, Hess and others. The use of mappings of monotone type allows one to treat problems with a certain lack of monotonicity and compactness.

The basic abstract example of mappings of monotone type is the duality map from a real reflexive Banach space into its dual space. The construction of the duality map shows that mappings of monotone type arise naturally from the structure of the Banach space. The duality map $J: X \rightarrow X^{*}$ is determined via the Hahn-Banach Theorem by the conditions

$$
\|J(u)\|=\|u\|, \quad\langle J(u), u\rangle=\|u\|^{2} \quad \text { for all } u \in X .
$$

By the strict convexity of $X^{*}$ the map $J$ is single valued. The duality map $J: X \rightarrow$ $X^{*}$ a homeomorphism, and it is homogeneous but not linear unless $X$ is a Hilbert space. Moreover, $J$ is strictly monotone and of class $\left(S_{+}\right)$(see [7, [1]).

The topological degree for mappings of class $\left(S_{+}\right)$is contructed by F. Browder [5] and V. Skrypnik [9]. An alternative construction is presented in [1, 4] and we recall it briefly here.

By the embedding theorem of Browder and Ton ([], see also []ㅡ) there exist a real separable Hilbert space $H$ and a compact linear injection $\phi: H \rightarrow X$ such that $\phi(H)$ is dense in $X$. We define a further 'adjoint' mapping $\hat{\phi}: X^{*} \rightarrow H$ by setting

$$
(\hat{\phi}(w) \mid v)_{H}=\langle w, \phi(v)\rangle \quad \text { for all } v \in H \text { and } w \in X^{*},
$$

where $(\cdot \mid \cdot)_{H}$ stands for the inner product of $H$. Then $\hat{\phi}: X^{*} \rightarrow H$ is a compact linear mapping, and since $\phi(H)$ is dense in $X, \hat{\phi}$ is also injective.

Let $G \subset X$ be an open bounded set and $F: \bar{G} \rightarrow X^{*}$ a bounded demicontinuous mapping of class $\left(S_{+}\right)$. We approximate $F$ by a Leray-Schauder-type mapping $F_{\lambda}$ : $\bar{G} \rightarrow X$ defined by

$$
F_{\lambda}=I+\lambda \phi \hat{\phi} F \quad \text { for any } \lambda>0 .
$$

Denote by $\mathrm{d}_{X}$ the degree for mappings of class $\left(S_{+}\right)$from $X$ to $X^{*}$. Assume that $0 \notin F(\partial G)$. It is easy to see that $0 \notin F_{\lambda}(\partial G)$ for all sufficiently large values of $l$. The topological degree $d_{X}(F, G, 0)$ is then defined by

$$
\mathrm{d}_{X}(F, G, 0)=\lim _{\lambda \rightarrow+\infty} \mathrm{d}_{L S, X}\left(F_{\lambda}, G, 0\right) .
$$

If $y \in X^{*} \backslash F(\partial G)$, then we define

$$
\mathrm{d}_{X}(F, G, y)=\mathrm{d}_{X}(F-y, G, 0) .
$$


It is shown in [4 that the above definition gives a classical topological degree normalized by $J$. The above construction is valid only if $F$ is bounded. However, the boundedness assumption can be removed by a simple trick. Indeed, let $F: \bar{G} \rightarrow X^{*}$ be a demicontinuous map of class $\left(S_{+}\right)$and $y \notin F(\partial G)$. It is easy to see that the set $F^{-1}(y) \cap G$ is compact and there exists an open set $D$ such that $F^{-1}(y) \cap G \subset D \subset G$ and $\left.F\right|_{D}$ is bounded. By the excision property of the degree, the value of $\mathrm{d}_{X}(F, D, 0)$ is independent of set $D$ as long as $F^{-1}(y) \cap G \subset D \subset G$ and $\left.F\right|_{D}$ is bounded. Hence we can define $\mathrm{d}_{X}(F, G, 0)$ to be the common value of all such $d_{X}(F, D, 0)$ (see [1] for details).

The degree $\mathrm{d}_{X}$ is unique and thus it coincides with the degree of Browder constructed via the Galerkin approximations and finite-dimensional Brouwer degree.

Let $V \subset X$ be a closed proper subspace, let $i_{V}: V \rightarrow X$ be the natural injection and let $i_{V}^{*}: X^{*} \rightarrow V^{*}$ be defined by the relation

$$
\left\langle i_{V}^{*}(w), v\right\rangle=\left\langle w, i_{V}(v)\right\rangle \quad \text { for all } v \in V \text { and } w \in X^{*} .
$$

By the embedding theorem of Browder and Ton there exist a real separable Hilbert space $W$ and a compact linear injection $\phi_{V}: W \rightarrow V$ such that $\phi(W)$ is dense in $V$. As earlier, the compact linear injective mapping $\hat{\phi}_{V}: V^{*} \rightarrow W$ is defined by the relation

$$
\left(\hat{\phi}_{V}(w) \mid v\right)_{W}=\left\langle w, \phi_{V}(v)\right\rangle \quad \text { for all } v \in W \text { and } w \in V^{*} .
$$

The degree $\mathrm{d}_{V}$ for bounded demicontinuous mappings of class $\left(S_{+}\right)$from $V$ to $V^{*}$ is then defined in analogy with (1.3) and (1.4). In the sequel we shall need a further map $\phi_{V}^{*}: X^{*} \rightarrow W$ defined by

$$
\left(\phi_{V}^{*}(w) \mid v\right)_{W}=\left\langle w, \phi_{V}(v)\right\rangle \text { for all } v \in W \text { and } w \in X^{*} .
$$

The linear map $\phi_{V}^{*}: X^{*} \rightarrow W$ is compact but not injective, since $\operatorname{Ker} \phi_{V}^{*}=\operatorname{Ker} i_{V}^{*}=$ $V^{\perp}=\left\{y \in X^{*} \mid\langle y, v\rangle=0\right.$ for all $\left.v \in V\right\}$, the annihilator of $V$. It is easy to see that $\phi_{V}^{*}=\hat{\phi}_{V} i_{V}^{*}$.

\section{The Reduction Theorem}

We are now ready to prove our main result. The notations are the same as in Section 1.

Theorem 2.1 (Reduction Theorem). Let $X$ be a real separable reflexive Banach space, $G \subset X$ an open bounded set and $F: \bar{G} \rightarrow X^{*}$ a demicontinuous map of class $\left(S_{+}\right)$. Assume that $0 \notin F(\partial G)$ and $V \subset X$ is a closed subspace such that

$$
\left(I-J^{-1} F\right)(\bar{G}) \subset V \text {. }
$$

Then

$$
d_{X}(F, G, 0)=d_{V}\left(\left.i_{V}^{*} F\right|_{V}, G \cap V, 0\right) .
$$

Proof. Assume first that $F$ is bounded. By condition (2.1) $F(\bar{G} \cap V) \subset J(V)$ and $F^{-1}(0) \cap G \subset V$. Moreover, if $u \in \bar{G} \cap V$ and $i_{V}^{*} F(u)=0$ in $V^{*}$, then $F(u) \in V^{\perp}$. On the other hand $F(u) \in J(V)$ and since $V^{\perp} \cap J(V)=\{0\}$, we have $F(u)=0$ in $X^{*}$. Hence

$$
F^{-1}(0) \cap \bar{G}=\left\{u \in \bar{G} \cap V \mid i_{V}^{*} F(u)=0\right\} .
$$

Especially $0 \notin i_{V}^{*} F(\partial(G \cap V))$, since $\partial(G \cap V) \subset \partial G \cap V$. Thus both degrees appearing in the statement of the theorem are well defined (it is clear that $\left.i_{V}^{*} F\right|_{V}: \bar{G} \cap V \rightarrow V^{*}$ 
is bounded, demicontinuous and of class $\left(S_{+}\right)$). By the definition of $\mathrm{d}_{X}$ there exists $\lambda^{\prime}>0$ such that

$$
\mathrm{d}_{X}(F, G, 0)=\mathrm{d}_{L S, X}\left(F_{\lambda^{\prime}}, G, 0\right)=\mathrm{d}_{L S, X}\left(I+\lambda^{\prime} \phi \hat{\phi} F, G, 0\right) .
$$

Denote $S_{\lambda^{\prime}}:=I+\lambda^{\prime} \phi_{V} \phi_{V}^{*} F: \bar{G} \rightarrow X$. Then by the definition of $\mathrm{d}_{V}$ we have

$\mathrm{d}_{V}\left(\left.i_{V}^{*} F\right|_{V}, G \cap V, 0\right)=\mathrm{d}_{L S, V}\left(\left.\left(I+\lambda^{\prime} \phi_{V} \hat{\phi}_{V} i_{V}^{*} F\right)\right|_{V}, G \cap V, 0\right)=\mathrm{d}_{L S, V}\left(\left.S_{\lambda^{\prime}}\right|_{V}, G \cap V, 0\right)$,

where we have used the fact that $\phi_{V}^{*}=\hat{\phi}_{V} i_{V}^{*}$. Now $S_{\lambda^{\prime}}: \bar{G} \rightarrow X$ is a LeraySchauder-type map and $\left(I-S_{\lambda^{\prime}}\right)(\bar{G}) \subset V$. In order to apply Thorem 1.1 we need to know that $0 \notin S_{\lambda^{\prime}}(\partial G)$ for a sufficintly large $\lambda^{\prime}$. Assume the contrary. Then we can find sequences $\left(\lambda_{k}\right)$ and $\left(u_{k}\right) \subset \partial G$ such that $\lambda_{k} \rightarrow \infty$ and

$$
u_{k}+\lambda_{k} \phi_{V} \phi_{V}^{*} F\left(u_{k}\right)=0
$$

for all $k \in \mathbb{Z}_{+}$. Clearly $\left(u_{k}\right) \subset V$. At least for subsequences we can write $u_{k} \rightarrow u \in V$ in $X$ and $F\left(u_{k}\right) \rightarrow w$ in $X^{*}$. It is not hard to see that $\phi_{V}^{*} w=0$, i.e., $w \in V^{\perp}$, and $\left\langle F\left(u_{k}\right), u_{k}\right\rangle \leq 0$. Hence $\langle w, u\rangle=0$ and thus

$$
\lim \sup \left\langle F\left(u_{k}\right), u_{k}-u\right\rangle \leq 0
$$

By the $\left(S_{+}\right)$-property of $F$ we get $u_{k} \rightarrow u \in \partial G \cap V$ and $w=F(u) \in V^{\perp}$. On the other hand $F(u) \in J(V)$ implying $F(u)=0$, which leads to a contradiction. Consequently, by Theorem 1.1

$$
d_{L S, V}\left(\left.S_{\lambda^{\prime}}\right|_{V}, G \cap V, 0\right)=d_{L S, X}\left(S_{\lambda^{\prime}}, G, 0\right) .
$$

The proof is complete as soon as we obtain the equality

$$
\mathrm{d}_{L S, X}\left(F_{\lambda^{\prime}}, G, 0\right)=\mathrm{d}_{L S, X}\left(S_{\lambda^{\prime}}, G, 0\right) .
$$

To prove (2.5) it is sufficient to show that taking $\lambda^{\prime}>0$ large enough,

$$
\left[(1-t) F_{\lambda^{\prime}}+t S_{\lambda^{\prime}}\right](u) \neq 0 \quad 0 \leq t \leq 1, \quad u \in \partial G .
$$

We shall argue by contradiction. Then there exist sequences $\left.\left(\lambda_{k}\right), t_{k} \in\right] 0,1[$ and $\left(u_{k}\right) \subset \partial G$ such that $\lambda_{k} \rightarrow \infty$ and

$$
\left(1-t_{k}\right) F_{\lambda_{k}}\left(u_{k}\right)+t_{k} S_{\lambda_{k}}\left(u_{k}\right)=0
$$

that is,

$$
u_{k}+\lambda_{k}\left[\left(1-t_{k}\right) \phi \hat{\phi} F\left(u_{k}\right)+t_{k} \phi_{V} \phi_{V}^{*} F\left(u_{k}\right)\right]=0
$$

for all $k \in \mathbb{Z}_{+}$. We obtain the key relation

$$
\left\langle F\left(u_{k}\right), u_{k}\right\rangle=-\lambda_{k}\left(1-t_{k}\right)\left\|\hat{\phi} F\left(u_{k}\right)\right\|_{H}^{2}-\lambda_{k} t_{k}\left\|\phi_{V}^{*} F\left(u_{k}\right)\right\|_{W}^{2},
$$

where $\|\cdot\|_{H}$ and $\|\cdot\|_{W}$ are the norms of $H$ and $W$, respectively.

At least for subsequences we have $u_{k} \rightarrow u \in X, t_{k} \rightarrow t \in[0,1]$ and $F\left(u_{k}\right) \rightarrow w \in$ $X^{*}$. Consequently, $(1-t) \phi \hat{\phi} w+t \phi_{V} \phi_{V}^{*} w=0$, implying

$$
(1-t)\|\hat{\phi} w\|_{H}^{2}+t\left\|\phi_{V}^{*} w\right\|_{W}^{2}=0 .
$$

If $t \neq 1$, then $w=0$ and if $t=1$, then $\phi_{V}^{*} w=0$, i.e., $w \in V^{\perp}$. To obtain a contradiction we consider two separate cases.

(a) Assume that $w=0$. Then by (2.6)

$$
\lim \sup \left\langle F\left(u_{k}\right), u_{k}-u\right\rangle \leq 0
$$

implying $u_{k} \rightarrow u \in \partial G$ and consequently $w=F(u)=0$, a contradiction. 
(b) Assume that $w \neq 0$. Then $t=1$ and $w \in V^{\perp}$. Denote $s_{k}=\left(1-t_{k}\right) \lambda_{k}$. By (2.6) we conclude that the sequence $\left(s_{k}\right)$ is bounded. Without loss of generality we can assume that $s_{k} \rightarrow s \geq 0$. Since $w \in V^{\perp}$,

$$
\langle w, u\rangle=\lim \left\langle w, u_{k}\right\rangle=-\lim s_{k}\left\langle w, \phi \hat{\phi} F\left(u_{k}\right)\right\rangle=-s\|\hat{\phi} w\|_{H}^{2} .
$$

Consequently, by (2.6),

$$
\lim \sup \left\langle F\left(u_{k}\right), u_{k}-u\right\rangle \leq \lim \sup \left[-s_{k}\left\|\hat{\phi} F\left(u_{k}\right)\right\|_{H}^{2}\right]+s\|\hat{\phi} w\|_{H}^{2}=0 .
$$

Hence $u_{k} \rightarrow u \in \partial G$ and consequently $w=F(u) \in V^{\perp}$. By assumption (2.1) $u-J^{-1} F(u) \in V$ and thus

$$
\langle F(u), u\rangle=\left\langle F(u), J^{-1} F(u)\right\rangle=\|F(u)\|^{2} \geq 0 .
$$

On the other hand

$$
\langle F(u), u\rangle=\langle w, u\rangle=-s\|\hat{\phi} w\|_{H}^{2} \leq 0 .
$$

Thus $F(u)=0$ with $u \in \partial G$, a contradiction. By (a) and (b) the proof is complete in case $F$ is bounded. Assume now that $F: \bar{G} \rightarrow X^{*}$ is demicontinuous and of class $\left(S_{+}\right)$, but not necessarily bounded. The set $F^{-1}(0) \cap G$ is compact, and there exists an open set $D$ such that $F^{-1}(0) \cap G \subset D \subset G$ and $\left.F\right|_{D}$ is bounded. By the excision property of the degree together with the known part of the result we thus get the desired result:

$$
\mathrm{d}_{X}(F, G, 0)=\mathrm{d}_{X}(F, D, 0)=\mathrm{d}_{V}\left(\left.i^{*} F\right|_{V}, D \cap V, 0\right)=\mathrm{d}_{V}\left(\left.i^{*} F\right|_{V}, G \cap V, 0\right) .
$$

Note that in a real separable Hilbert space $X$, where $X^{*}=X$, the duality map is the identity, and the crucial condition (2.1) takes a more familiar form $(I-F)(\bar{G}) \subset V$.

\section{REFERENCES}

[1] J.Berkovits, On the degree theory for mappings of monotone type, Ann.Acad.Sci.Fenn.Ser.A1, Dissertationes, 58 (1986). MR0846256 (87f:47084)

[2] J.Berkovits, Topological degree theory and multiplication theorem for a class of nonlinear mappings, Bull.London Math.Soc. (23) (1991) pp. 596-606. MR.1135193 (92k:47118)

[3] J.Berkovits, A note on the imbedding theorem of Browder and Ton, Proc. AMS 131 (9) (2003) pp. 2963-2966. MR1974355 (2004b:46021)

[4] J.Berkovits and V.Mustonen, On the topological degree for mappings of monotone type, Bull. Amer. Math. Soc. (9) 10 (1986) pp. 1373-1383. MR0869546 (88b:47073)

[5] F.E.Browder, Fixed point theory and nonlinear problems, Proc. Sympos. Pure Math. (39) Part 2, AMS, Providence R.I, 1983 pp. 1-39. MR.0699315 (84h:58027)

[6] F.E.Browder and B.A.Ton, Nonlinear functional equations in Banach spaces and elliptic super-regularization, Math.Z. 105 (1968) pp. 177-195. MR0232256 (38:582)

[7] K.Deimling, Nonlinear functional analysis, Springer-Verlag, Berlin, 1985. MR0787404 (86j:47001)

[8] N.G.Lloyd, Degree Theory, Cambridge University Press, Cambridge, 1978. MR0493564 $(58: 12558)$

[9] I.V.Skrypnik, Topological methods of investigations of operator equations and nonlinear boundary value problems, Nonlinear Analysis, Function Spaces and Applications, TeubnerTexte zur Mathematik 49 (1982) pp. 191-234. MR0685001 (84e:58069)

[10] E.Zeidler, Nonlinear Functional Analysis and its Applications I, Fixed-Point Theorems, Springer-Verlag, New York, 1985. MR0816732 (87f:47083)

[11] E.Zeidler, Nonlinear Functional Analysis and its Applications II/B, Nonlinear Monotone Operators, Springer-Verlag, New York, 1985. MR0768749 (90b:49005) 
Department of Mathematical Sciences, University of Oulu, P.O. Box 3000, Fin-90014 Oulu, Finland

E-mail address: juha.berkovits@oulu.fi 